\title{
Mid-infrared narrow band plasmonic perfect absorber for vibrational spectroscopy
}

\author{
Semih Korkmaz ${ }^{\mathrm{a}, \mathrm{b}}$, Mustafa Turkmen ${ }^{\mathrm{a}, \mathrm{c}}$, Serap Aksu ${ }^{\mathrm{b}, \mathrm{d}, *}$ \\ a Department of Electrical and Electronics Engineering, Erciyes University, Kayseri 38039, Turkey \\ ${ }^{\mathrm{b}}$ UNAM-Institute of Materials Science and Nanotechnology, Bilkent University, Ankara 06800, Turkey \\ ${ }^{c}$ Fotonik Technology and Engineering Co. Inc., Erciyes Technopark Co., Kayseri 38010, Turkey \\ ${ }^{\mathrm{d}}$ Department of Physics, Koc University, Istanbul 34450, Turkey
}

\section{A R T I C L E I N F O}

\section{Article history:}

Received 22 August 2019

Received in revised form

11 November 2019

Accepted 14 November 2019

Available online 16 November 2019

\section{Keywords:}

Narrow-band perfect absorber

Nanoantenna

Multi-band resonances

Mid-infrared

Surface enhanced infrared absorption

(SEIRA) spectroscopy

Metal-insulator-metal systems

\begin{abstract}
A B S T R A C T
The introduction of narrow band perfect absorbers (PAs) operating in mid-infrared (IR) frequencies has the potential to improve the study of biomolecule monolayers using surface enhanced vibrational spectroscopy. However, wavelength dependent radiation and nonradiative losses are limiting factors to obtain narrow band absorption resonance, having full width at half maximum (FWHM) below $150 \mathrm{~cm}^{-1}$ in the mid-IR frequency range. In this work, we numerically and experimentally present an engineered narrow band PA with FWHM of $85 \mathrm{~cm}^{-1}$ operating in mid-IR frequencies. The PA is based on multi-layer metamaterial plasmonic nanoantennas. We used numerical and experimental work to fine tune the parameters of the PA in order to control its spectral response. The suggested PA system strongly provides high absorption values (100\%) in the mid-IR region and near-field enhancement factor of $10^{3}$ at the corresponding resonance values. The fabricated PA shows strong narrow band resonance and all the resonance energy could be transferred to thin films $(10 \mathrm{~nm})$ for higher sensitivity. In addition, PA shows multi-band resonances within the mid-IR region, thus it can be effectively used for simultaneously detecting the different biomolecular fingerprints. In this sense, we experimentally observed absorption of carbonyl $v(\mathrm{C}=0)$ and asymmetric methyl $v\left(-\mathrm{CH}_{3}\right)$ stretching bands of thin polymethyl methacrylate (PMMA) film on the narrow band resonances of PA. The suggested system has potential to be used in live cell-membrane investigations, as multi-band structure enables investigation of both lipids and proteins.
\end{abstract}

(c) 2019 Elsevier B.V. All rights reserved.

\section{Introduction}

Among many optical biosensing techniques vibrational spectroscopy has been instrumental as it is capable of both identification and detection of molecules [1-3]. This technique is based on the fact that molecule-specific vibrations occur in mid-infrared (IR) range (3-8 $\mu \mathrm{m}$ wavelengths) [4]. In this process, light is absorbed by the dipolar molecular bond at certain frequencies, and this absorption can be tracked to identify the molecules and molecular structures/orientations [5]. Surface enhanced infrared absorption (SEIRA) spectroscopy technique utilizes this approach for sensitive characterization of even few biomolecules [6]. Surface enhancement is provided by active plasmonic surfaces that act as an antenna which can direct the light to a biomolecule (lipid, protein, polymer,

\footnotetext{
* Corresponding author at: Department of Physics, Koc University, Istanbul 34450, Turkey.

E-mail address: saksu@ku.edu.tr (S. Aksu).
}

etc.) at its specific absorption frequency. Thus, the energy radiated by the nanoantennas could be transferred to the biomolecule for enhancing the absorption signal intrinsic to molecular bonds [7-13]. The intensity of the directed light, also called the enhanced near electric field (E), is proportional to the absorption signal that arises from the biomolecule [14,15]. This implies that when the resonance band of the plasmonic nanoantenna is narrow, we could transfer more resonance energy towards the biomolecule, precisely at the absorption frequency of the molecular bonds [15]. That results in more significant absorption signal generation from the same number of molecules, thus higher sensitivity can be obtained by a narrow band resonance nanoantenna [16-18]. In addition, using narrow band plasmonic resonances, we can obtain resolutions that can probe each specific molecular absorption frequencies, even if they are separated only by $100 \mathrm{~cm}^{-1}$. The IR vibrational fingerprints provide intrinsic information on the molecular structures of biomolecules and enable detection and more importantly identification of them. For instance, the amide I fingerprint of a protein contains conformational information that is crucial for understand- 
ing its function. Using the narrow-band antennas, one can also detect and sense the presence of such molecules, as well as acquire special information intrinsic to biomolecule [7].

Metamaterial PAs opened new doors to the field of optical energy harvesting devices. By providing narrow band plasmonic resonances, PAs have the potential to further improve the surface enhanced vibrational spectroscopy platforms. PAs can be typically designed using three functional layers that comprise a dielectric interlayer sandwiched between the bottom metal film and a patterned top metal plasmonic nanoantenna serving as a resonator [19]. Three-layered configuration includes an important parameter, dielectric layer thickness that allows to bring the absorption level to unity (100\%) and width of the resonance down to $85 \mathrm{~cm}^{-1}$ (Quality factor, $Q=\lambda / \Delta \lambda=22$ ). The top layer of PAs which are subwavelength plasmonic antenna components, absorb most of the incident radiation at the operating frequency. In earlier reports of metamaterial PAs, near unity absorption was explained over a narrow frequency range by minimizing the reflectance with perfect impedance matching $(\mathrm{z}=\sqrt{\mu / \varepsilon}=1$, complex electric permittivity $(\varepsilon)$ and magnetic permeability $(\mu)$ ) while eliminating the transmittance with the metal film [19]. Engineered PAs can create intense confined hotspots of the incident light which provide strong interaction with adjacent analytes and make them ideal candidates for biosensing and spectroscopy applications [20-22]. Resonance frequencies of these structures can be tuned to molecular resonance frequencies of biomolecules and polymers in mid-IR [10,23]. Functionalization of PAs with molecules results in coupling plasmonic resonances with molecular absorption [24]. This coupling supports Fano line shapes that occur in the presence of interference between one spectrally broad and one narrow energy state [25-28]. Molecular bands of polymers have narrow band infrared spectral features [29]. By designing narrow band metasurfaces, we can reach these molecular bands precisely one by one.

In this work, we present a new narrowband metamaterial PA operating in the mid-IR frequency regime that is relevant to analysis of biomolecules and demonstrate the usage of a narrowband PA for sensing with vibrational spectroscopy for the first time. The structure is composed of multiple, stair-like changinglength rod antennas placed horizontally and vertically, as shown in Fig. 1(a). We analyze the nanoantenna design in finite difference time domain (FDTD) based commercial software Lumerical FDTD Solutions [30]. We analyze geometrical parameter sensitivity of the structure by changing the lengths of the nanorods finely to tune the resonance frequency to molecular absorption frequencies of polymethyl methacrylate (PMMA). At resonance frequencies, PA platform shows high near-field intensity enhancement $\left(\left|E^{2}\right| /\left|E_{\text {incident }}{ }^{2}\right|\right)$ with large area of hotspots which are indispensable to improve the light-matter interaction. In experimental study, an optically thick gold $(\mathrm{Au})$ film and an ultra-thin (< $\lambda / 50)$ magnesium fluoride $\left(\mathrm{MgF}_{2}\right)$ spacer are coated orderly on silicon $(\mathrm{Si})$ to prepare multilayered metamaterial perfect absorbers. By using standard e-beam lithography and lift-off processes, top metallic nanoantennas are formed on $\mathrm{MgF}_{2}$ layer. We use a Fourier transform infrared (FTIR) spectrometer coupled to an IR microscope to obtain the absorption spectrums of the fabricated platforms. Once we tune the antenna resonance to the molecular absorption frequencies of PMMA, we experimentally place a $10 \mathrm{~nm}$-thick PMMA film in the close vicinity of the PAs and match the plasmonic resonances of the PA with carbonyl $(\mathrm{C}=\mathrm{O})$ stretch band $(1729$ $\left.\mathrm{cm}^{-1}\right)$ and asymmetric methyl $\left(-\mathrm{CH}_{3}\right)$ stretch band $\left(2952 \mathrm{~cm}^{-1}\right)$ of the PMMA thin film. Using the narrow band metamaterial PA, we obtained very high molecular absorption signals from PMMA compared to prior reported PA's. Chen et al. [31] demonstrated experimental dual-band PA in the mid-IR regime. In that work, experimental FWHM values of dual-band resonances are signif- icantly broader $\left(167.5 \mathrm{~cm}^{-1}\right)$ compared to our results, thus the PMMA absorption signal observed on the antenna resonance is barely visible. Chen et al. used only $4 \mathrm{~nm}$ thick PMMA layer (in comparison to $10 \mathrm{~nm})$, still the measured asymmetric methyl $\left(-\mathrm{CH}_{3}\right)$ stretch band signal has been significantly lower compared to the signal obtained by our narrow-band perfect absorber ( $1.5 \%$ vs 0.5 $\%$ absorption unit). Recently, Durmaz et al. [32] experimentally designed a broadband PA for detecting PMMA thin layer. However, they did not report the asymmetric methyl stretch band signal, presumably because the signal could not be detected with their perfect absorber. This comparison reflects that the narrow band PA's can significantly improve the surface enhanced IR absorption spectroscopy.

\section{Materials and methods}

\subsection{Numerical and experimental analysis of the designed PA}

In this section, we explain the specific geometry of the resonant plasmonic perfect absorber. Top and cross-sectional view of the dual-resonant PA are shown in Fig. 1(a) and (b), respectively. The PA consists of an array of combined plasmonic rods separated from an optically thick non-transparent metallic ground plate. In the optimum configuration, we use a $100 \mathrm{~nm}$-thick $\mathrm{MgF}_{2}$ spacer between a patterned $80 \mathrm{~nm}$-thick Au nanoparticle-based top layer and a $200 \mathrm{~nm}$-thick Au ground plate. The whole sensing platform is designed on a silicon substrate with a thickness of $500 \mathrm{~nm}$. Polarization of the illumination source is along the $x$-direction. The frequency-dependent dielectric constants of the metals are taken from Palik [33], and optical constants of $\mathrm{MgF}_{2}$ dielectric spacer are taken from Dodge [34]. We determine lengths of rods as $L_{1}=2600 \mathrm{~nm}, L_{2}=2400 \mathrm{~nm}, L_{3}=2200 \mathrm{~nm}, L_{4}=2000 \mathrm{~nm}$, $L_{5}=840 \mathrm{~nm}, w_{1}=400 \mathrm{~nm}, w_{2}=200 \mathrm{~nm}$, and the array periodicities $\left(P_{\mathrm{x}}=P_{\mathrm{y}}\right)$ are $3.2 \mu \mathrm{m}$. We fabricate the PAs via electron-beam lithography (EBL) and a following lift-off process. Firstly, the $200 \mathrm{~nm}$-thick Au film and $100 \mathrm{~nm}$-thick $\mathrm{MgF}_{2}$ dielectric spacer are deposited onto the Si substrate by using electron-beam evaporation. Secondly, the $200 \mathrm{~nm}$-thick PMMA is coated as a positive photo resist on top of the $\mathrm{MgF}_{2}$ layer by spin-coating. EBL is performed on PMMA. Then, EBL-defined patterns are removed by passing the chip through methyl isobutyl ketone-isopropyl alcohol (MIBK-IPA (1:3)) solution for $50 \mathrm{~s}$. EBL-exposed parts are then covered with 5 nm-thick titanium (Ti) as an adhesion layer and $80 \mathrm{~nm}$-thick Au by e-beam evaporation, respectively. Finally, the standard lift-off process is applied to obtain nanoantennas. Fabricated PA samples are characterized optically by a commercial Bruker Hyperion 2000 FTIR microscope that has a $15 x$ Cassegrain objective $(N A=0.4)$ to collect reflection signals during the measurements. Due to the two-mirror configuration of the objective some part of the light may impinge on the sensor surface with up to $12^{\circ}$ angle. The discussion in Fig. S1 (see Appendix A: Supplementary Information S1) shows that the effect of small incident angle change is tolerable for the experiments, and the experimental results still agree well with the simulations, especially for the first mode of PA. Absorption data is normalized via an optically thick Au film. Fig. 1(c) shows numerical and experimental absorption spectra of the PA. Numerical and experimental absorption spectra are in well agreement for the first mode at smaller wavenumbers in the terms of resonance frequency and absorption value. For the second mode at higher wavenumber, we obtain nearly the same resonance frequency and lower intensity. The full width at half maximum (FWHM) for experimental results are surprisingly narrower than the simulation results. This effect is still under investigation; however, we think it can be explained by the curved corners of the fabricated structures compared to the sharp edges used in the simulations [22,23]. We call this PA as a narrow band PA since previous studies on multi-mode multilayer PA's [31] 
(a)

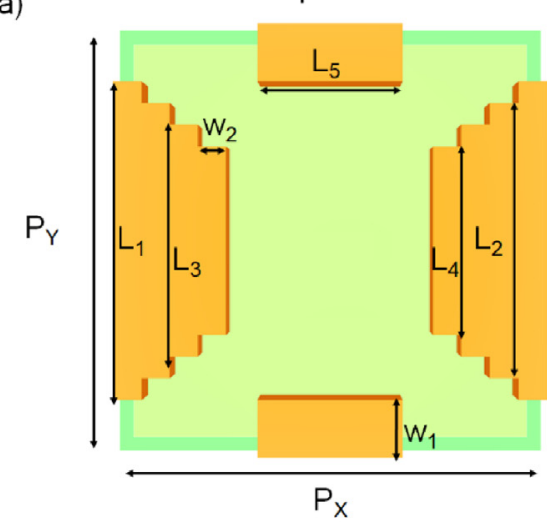

(c)



(b) Cross section<smiles></smiles>

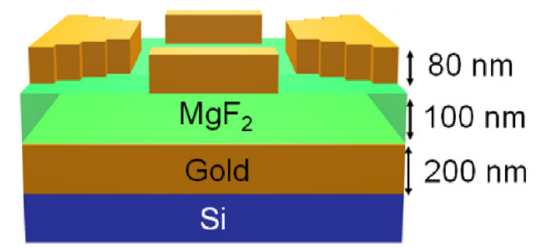

(d)

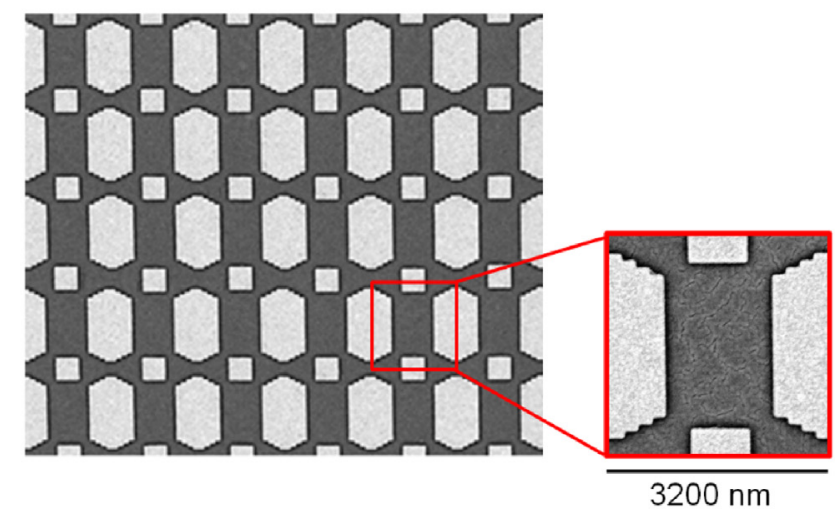

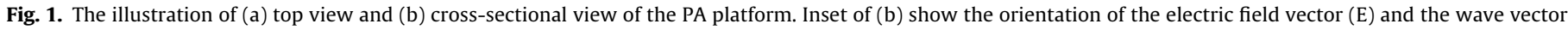


fabricated PA arrays. The PA unit cell is in the red square.

regularly provided considerably broader $\left(167.5 \mathrm{~cm}^{-1}\right)$ FWHM values in the mid-IR regime. The PA platform supports two resonance modes; the first mode is observed at around $v_{1}=1830 \mathrm{~cm}^{-1}$ with more than $90 \%$ absorption. The deviation from unity absorption could be explained with usage of a Cassegrain objective where some part of the incident light fall on sensor surface with a small $\left(\sim 10^{\circ}\right)$ angle. The second mode is obtained at $v_{2}=2810 \mathrm{~cm}^{-1}$ with $70 \%$ absorption, while numerical results show $90 \%$. This is because the electromagnetic coupling is not strong enough between the vertical and horizontal rods in the unit cell. This effect will be explained further in Section 3.3. We critically tune the resonances to $v_{1}$ and $v_{2}$ to target the absorption frequency of the molecular bonds of interest. Fig. 1(d) presents the scanning electron microscope (SEM) image of the fabricated PAs. The surface of the PA is smooth, and the unit cell is defined in the red box.

We design the simulation platform identical to the configuration that we take the spectroscopic measurements. Thus, the light illumination has been a plane wave, sent normally on the structures. There is no angle introduced on the IR light illumination, both in measurements and in simulations. From the simulations we know that the structures are polarization dependent, so we applied the same polarization direction that is used in the simulations to the IR illumination in the microscope. We have analyzed the effect of periodicity of PA, the effect of polarization angle and angle of incidence using simulations (see Appendix A: Supplementary Information $S 1)$. As the angle of incidence deviates from normal, the perfect absorption $(A=1)$ is lost and resonance intensity decreases. Similarly, when the polarization angle is changed from zero (respect to $\mathrm{x}$-coordinate) to 30 degrees, the perfect absorption is lost. When we increase periodicity, there is no significant change for the first resonance mode, but the second resonance slightly red-shifts.

\section{Results and discussions}

\subsection{Comparison of full width at half maximum (FWHM) values for particle and PA based nanoantennas}

The narrowing on the resonance of the proposed PA is compared with the configuration where a geometrically identical antenna design is placed on top of the dielectric substrate as seen in Fig. 2(a). When the same top antenna design is placed on a $100 \mathrm{~nm}-$ thick $\mathrm{MgF}_{2}$ substrate, we observe very broad reflection resonances. The numerical and experimental results clearly show that the PA has narrower resonances and higher absorption when a dielectric spacer with finite thickness is sandwiched between ground metal plate and top metal antenna layer in Fig. 2(b) and (c), respectively. The dielectric spacer has the maximum impact on the perfect absorption as shown in Fig. 2(d). The prior simulation results revealed that the perfect absorption $(A=1)$ can be achieved at various spacer thickness periodically at certain operation frequencies $[31,35]$. When the thickness is low $(<200 \mathrm{~nm})$ strong coupling between the top antenna and ground plate occurs. The thickness of the spacer has to be tuned critically to provide destructive interference of light that is directly reflected from the top antenna layer and reflections occurring in the spacer. In Fig. 2(d), we have also shown that the spacer thickness has to be tuned carefully in order to obtain near unity absorption. Any change of the spacer thickness slightly affects the FWHM, but the absorption changes dramatically.

\subsection{The physical origin of the resonance frequencies}

We analyze the near-field enhancement distributions to learn the physical origin of the resonance frequencies through FDTD 
(a)

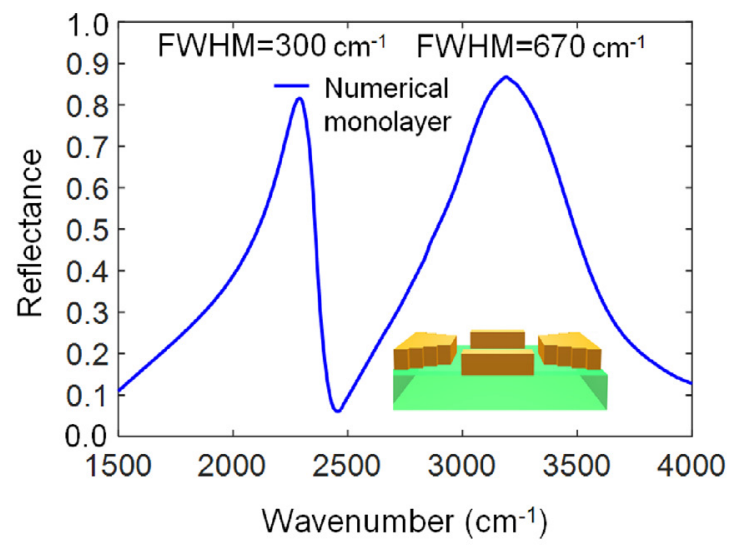

(c)



(b)

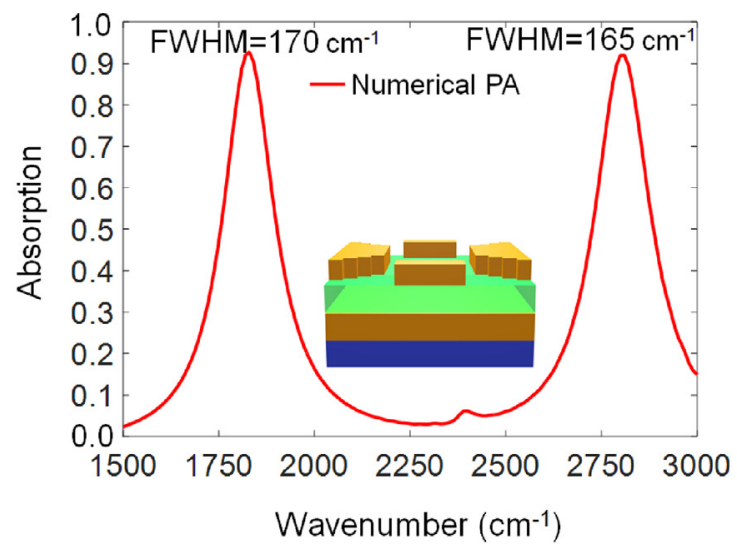

(d)

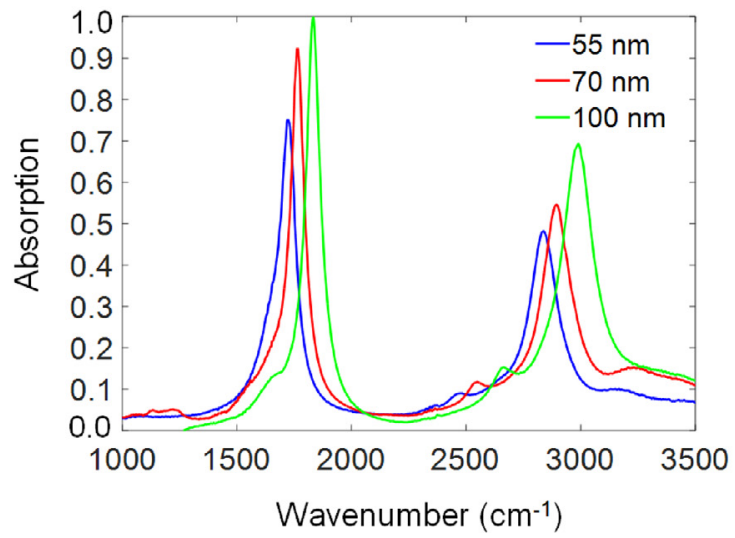

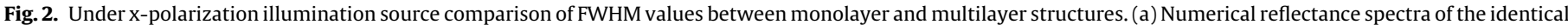

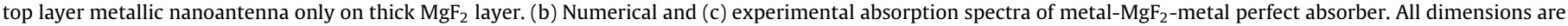


has to be tuned carefully to obtain narrow band resonances and near unity absorption.

simulations. Fig. 3(a) shows calculated reflectance (R), transmittance (T), and absorption (A) spectra of the PA design in the mid-IR regime for the values of $L_{1}=2600 \mathrm{~nm}, L_{2}=2400 \mathrm{~nm}, L_{3}=2200 \mathrm{~nm}$, $L_{4}=2000 \mathrm{~nm}, L_{5}=840 \mathrm{~nm}, w_{1}=400 \mathrm{~nm}, w_{2}=200 \mathrm{~nm}$, and the array periodicity is $3.2 \mu \mathrm{m}$. Absorption is defined as $A=1-R-T$ [31]. In order to achieve perfect absorption $(A=1)$, one should minimize the reflection and transmission. The transmission over the structures is zero as the light is fully blocked by the optically thick Au film. Since the metal ground plate is optically thick and not transparent, the absorption here can be defined as $A=1-R$. The perfect absorption can be explained using critical coupling [36,37], meaning that the rate of radiative loss or leakage rate of energy out of the resonator is equal to dissipative loss [38,39]. Maximum coupling can be obtained by matching the incident electric field pattern to the radiation pattern of a localized surface plasmon resonance, and light scattering in whole directions is eliminated [40,41]. The elimination of scattering means that the reflection is now reduced to zero, thus $A=1$. The obtained reflectance and absorption spectra have dual-band resonances, due to the fact that the incident radiation mainly interacts with two different types of nanorods that are located at $\mathrm{x}$ - and $\mathrm{y}$ - axes at the same time.

Fig. 3(b) presents calculated near-field intensity enhancement values for the proposed PA at the mid-IR regime. After obtaining resonance frequencies of the structure at the maximum absorption, we have calculated near-field enhancement distributions and values via the same simulation program. We place vertical and hor- izontal field monitors between $\mathrm{MgF}_{2}$ film and nanoantenna where the energy is enhanced. When we use horizontal field monitors, we obtain near-field enhancement distributions and values in Fig. 3(b). The enhancement factor is 3800 at the first mode $\left(v_{1}=1700 \mathrm{~cm}^{-1}\right)$, and 5800 at the second mode $\left(v_{2}=2910 \mathrm{~cm}^{-1}\right)$. It is observed that the first resonant mode originates from the vertical nanorods, and the second from the horizontal ones. Along the mid-IR regime, enhancement values never reach zero due to the "lightning-rod effect" [42]. In order to calculate and visualize cross-sectional nearfield distributions, we utilize vertical field monitors. The amplitude value of the incident field is 1 . Fig. $3(\mathrm{c}-\mathrm{f})$ show the calculated cross-sectional profiles of the near field intensity under $\mathrm{x}$-polarized illumination source for the modes at $v_{1}$ and $v_{2}$ for electric and magnetic responses, respectively. Fig. 3(c) and (d) show total electric field intensity enhancement $\left(\left|E^{2}\right| /\left|E_{\text {incident }}{ }^{2}\right|\right)$ and distributions for the first and second mode, respectively. Highly enhanced local electric fields are mainly concentrated at the vertical and horizontal nanorods. Fig. 3(e) and (f) show total magnetic field intensity $\left(\left|\mathrm{H}^{2}\right| /\left|\mathrm{H}_{\text {incident }}{ }^{2}\right|\right)$ and distributions for the first and second modes, respectively. As it is seen in the Fig. 3(e) and (f), magnetic fields are mostly presented between $\mathrm{MgF}_{2}$ and Au antenna border area due to the nature of multilayer structure. We obtain higher magnetic field values compared to the reported perfect absorbers [43]. At each resonance frequency, localized surface plasmons (LSPs) are supported by strong absorption at optical wavelengths. Hence, large near-field confinement can enable us to obtain high-throughput light-matter 


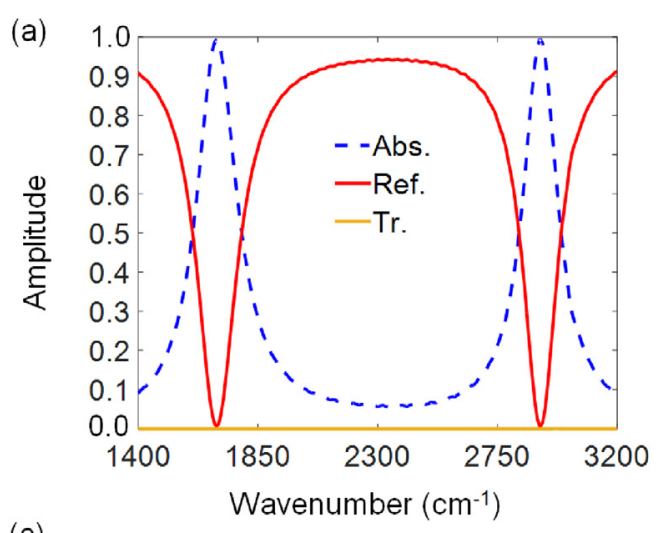

(c)


(b)

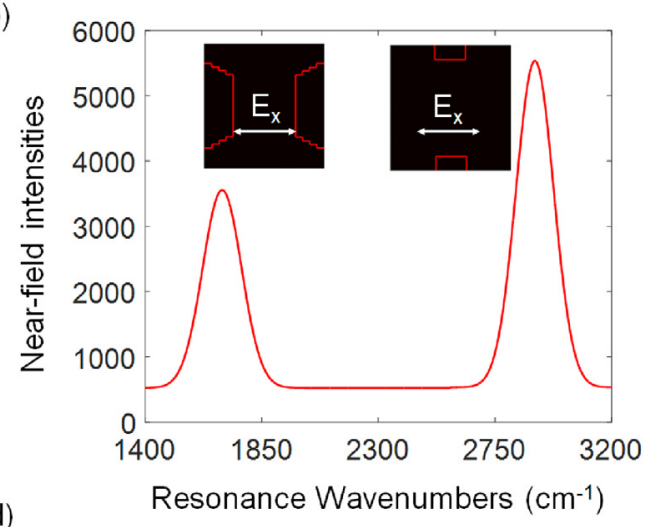

(d)
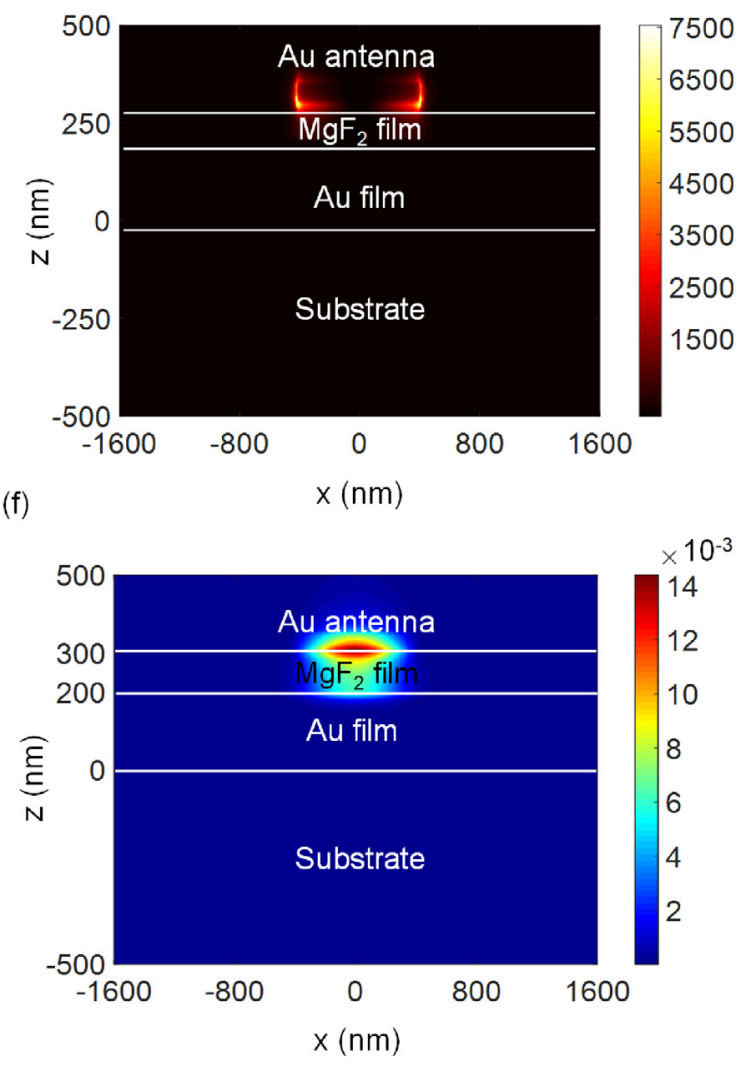

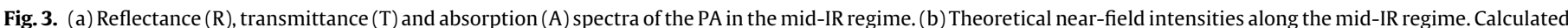

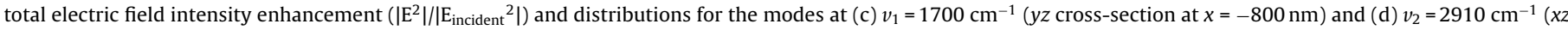

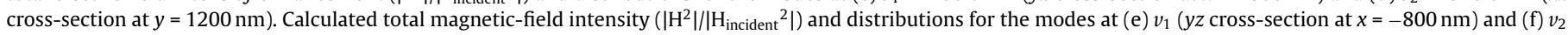
( $x z$ cross-section at $y=1200 \mathrm{~nm}$ ).

interaction applications such as simultaneous identification of different molecular fingerprints.

\subsection{Dependence of the spectral response on parameter sweep}

To demonstrate the fine-tuning mechanism, we have changed lengths of both vertical and horizontal nanorods in the unit cell of the PA. High parameter sensitivity of the suggested PA easily enables us to reach exact molecular resonance frequencies of polymers or biomolecules. In order to achieve easy tunability in a broader frequency range, we use a step-down structure instead of using a single rod with the same width. With the step-down structure we can enable broader resonance frequency tunability compared to the use of single rod. When we change $\mathrm{L}_{1}$ from $2600 \mathrm{~nm}$ to $2400 \mathrm{~nm}$, the change of resonance is broader for the step-down structure. There is no significant change in the resonance frequency at the first mode for the no stepping-down structure (see Appendix A: Supplementary Information S2). More resonance shift by an incremental change in the length means that the step-down structure is more easily tunable over a broader frequency range, which is desired for surface enhanced IR spectroscopy. Fig. 4 shows the calculated and experimental parameter sweeps for $L_{1}$ and $L_{5}$. For both numerical and experimental analyses, the length of the vertical rods $\left(L_{1}, L_{2}, L_{3}\right.$ and $\left.L_{4}\right)$ in the unit cell is reduced by $200 \mathrm{~nm}$. Fig. 4(a) presents calculated absorption spectra for different $L_{1}$ values. As $L_{1}$ increases, the first mode slightly red-shifts under $\mathrm{x}$-polarized illumination source. The second absorption peak does not change its magnitude or does not change the resonance peak frequency under the same illumination. Fig. 4(b) shows the dependence of the calculated absorption 
(a)

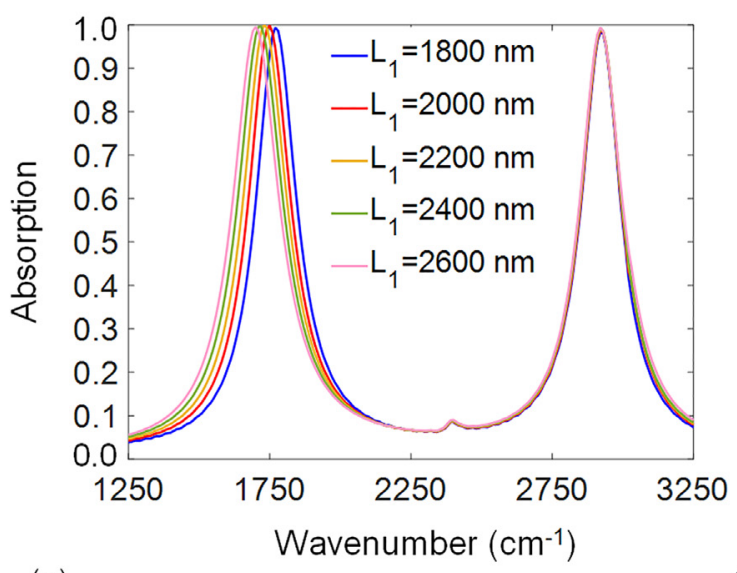

(c)

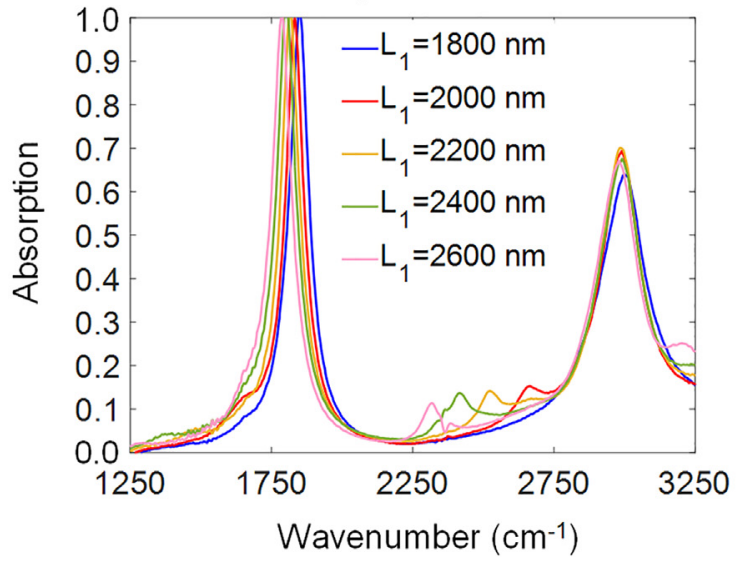

(b)

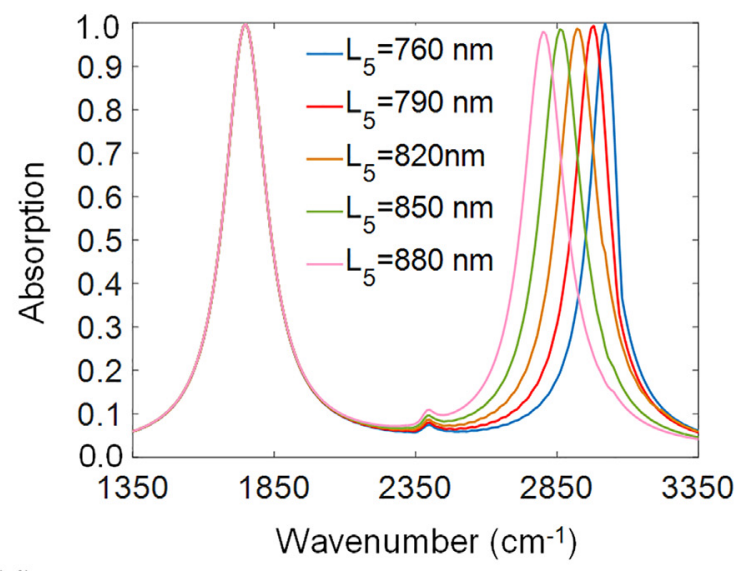

(d)





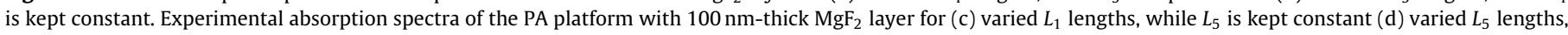

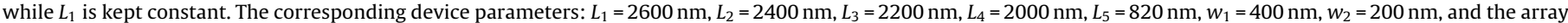
periodicities $\left(P_{\mathrm{x}}=P_{\mathrm{y}}\right)$ are $3.2 \mu \mathrm{m}$.

spectra on $L_{5}$. As $L_{5}$ is increased, the second mode significantly red shifts. The first resonance mode does not change when $L_{5}$ value is changed. In Fig. 4(c), experimental absorption spectra can be seen for different $L_{1}$ values. As $L_{1}$ increases, the red-shifting behavior of the first resonance mode in calculations agrees well with experimental results, under x-polarization. The behavior of the second resonance mode matches the calculations well in terms of the resonance peak frequency. Fig. 4(d) shows the dependence of the experimental absorption spectra on $L_{5}$. As $L_{5}$ increases, the first mode agrees well with the calculated results. Even though all experimental results agree well with the calculations, there is a difference on the intensity of the second resonance mode. We believe that the lower intensity obtained at the second resonance mode of the fabricated PA is due to the dielectric spacer thickness and microscopic defects occurring on the surface of spacer. We experimentally showed that when the thickness of $\mathrm{MgF}_{2}$ increases, the resonance intensities of the first and second modes also increase (Fig. 2(d)). For some PAs, after $\mathrm{MgF}_{2}$ deposition we have observed bubbles in the film. Given that spacer thickness is critical for perfect absorption, we think that the surface roughness and small defects on the $\mathrm{MgF}_{2}$ film affect the second resonance mode. The experimental and numerical results confirm the electromagnetic field distributions. We observe an additional small peak at $2300 \mathrm{~cm}^{-1}$ in both numerical and experimental results. In the simulations, we have confirmed that this small peak arises from the Ti adhesion layer (see Appendix A: Supplementary Information S3). As it is seen in Fig. 4(c) and (d), the position of the Ti peak is not fixed. During the fabrication the intended Ti film thickness may vary couple of nm's with respect to the Ti thickness in simulations, and this causes a shift on the Ti peak. The most striking contrast between the calculated and experimental optical responses is that the fabricated PA has narrower FWHM compared to the calculations. While the FWHM value is $170 \mathrm{~cm}^{-1}$ for the calculated result, the experimental measurements show this value as $85 \mathrm{~cm}^{-1}$. This effect is highly desirable for detecting the vibrational modes of polymers due to the fact that these vibrational modes mostly show narrow band feature. As previously stated, we think this effect happens due to the curved corners and edges of the fabricated structures compared to the sharp ones in simulations. The suggested narrow band plasmonic PAs provide more successful vibration band matching compared to the monolayer plasmonic antennas since they have a higher resonance quality factor, and thus can provide better sensitivity.

\subsection{Experimental sensing capacity of the PA and the importance of matching the plasmonic resonance with molecular absorption bands}

To demonstrate the capacity of the suggested narrow-band PA in surface enhanced infrared absorption spectroscopy, we performed simultaneous detection of two specific and spectrally far vibration modes of a PMMA film experimentally. Plasmonic resonances decay 




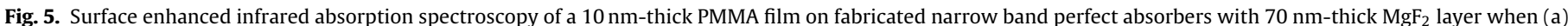



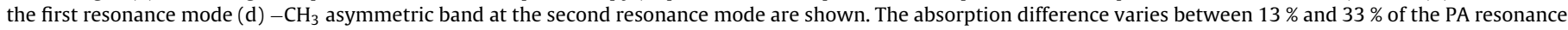
intensity for the $\mathrm{C}=\mathrm{O}$ band and $1.5 \%$ for the $-\mathrm{CH}_{3}$ asymmetric band.

away from the surface of the metal and they are active up to $100 \mathrm{~nm}$ above the surface $[44,45]$. Thus, we coated a $10 \mathrm{~nm}$-thick PMMA film by spin-coating ( $5000 \mathrm{rpm}, 25 \mathrm{~s}$.). Thickness of the PMMA film is characterized using J. A. Woollam Co. VASE ellipsometer (see Appendix A: Supplementary Information S4). Control measurements are performed on an Au coated $\mathrm{MgF}_{2}$ film. No molecular absorption signals are obtained for the same PMMA film thickness on the control measurements. Fig. 5(a) and (c) present experimental absorption spectra of the PA platform with 70 nm-thick $\mathrm{MgF}_{2}$ layer after being coated by PMMA film. The PA parameters $L_{1}$ and $L_{5}$ that overlaps with the PMMA absorption peaks are determined as $2400 \mathrm{~nm}$ and $790 \mathrm{~nm}$, respectively. Absorption is more evident when $L_{1}$ increases since the overlap of carboxyl bond with PA resonance is maximum. As it is seen in the Fig. 5 (a), when we match plasmonic resonance frequency $\left(1729 \mathrm{~cm}^{-1}\right)$ with the molecular absorption frequency of carbonyl bands, PMMA starts to absorb more energy from nanoantenna which offers the maximum enhanced field available. The carbonyl $v(\mathrm{C}=0)$ stretch band frequency is observed at $1729 \mathrm{~cm}^{-1}$ and asymmetric methyl $v\left(-\mathrm{CH}_{3}\right)$ stretch band is observed at $2952 \mathrm{~cm}^{-1}$. The absorption intensity of the carbonyl $(\mathrm{C}=\mathrm{O})$ band on the PA resonance is stronger than the absorption intensity of $-\mathrm{CH}_{3}$ asymmetric band. When we look at the attenuated total reflection (ATR) spectroscopy result of the PMMA thin film (see Appendix A: Supplementary Information S5), we see that amount of absorbed energy is higher for carbonyl bond in comparison with the $\mathrm{C}-\mathrm{H}$ bonds. Thus, observ- ing a strong absorption signal from carbonyl is expected. Finally, we experimentally perform surface enhanced infrared absorption spectroscopy for all PA arrays in order to show the importance of matching narrow band resonances with molecular absorption bands. Absorption dip for the $\mathrm{C}=\mathrm{O}$ stretch band (around $1729 \mathrm{~cm}^{-1}$ ) can be clearly seen for all PAs in Fig. 5(a). When $L_{5}$ is equal to $790 \mathrm{~nm}$, we obtain the strongest absorption signal of the $-\mathrm{CH}_{3}$ asymmetric band stretch at $1752 \mathrm{~cm}^{-1}$ in Fig. 5(c). In Fig. 5(b) and (d), experimental difference spectroscopy of PMMA is shown. This data is obtained from the PMMA coated PA that has 70 nm-thick MgF2 layer. The experimental spectra of PMMA coated PA is subtracted from the uncoated PA's experimental spectra (see Appendix A: Supplementary Information $\mathrm{S} 6$ ) for the $\mathrm{C}=\mathrm{O}$ band (red line) and the $-\mathrm{CH}_{3}$ asymmetric band (blue line), respectively. After PMMA binding no red-shift is observed on the PA resonance for the first mode (see Appendix A: Supplementary Information S6), thus we applied direct subtraction without correcting the shift with a polynomial fitting [44]. In order to reliably calculate the absorption difference ( $\triangle$ Absorption $=A_{\text {fit }}-A_{\text {PMMA }}$ ) in the presence of the spectral shifts for the second mode, we employ the fitting procedure [44] which shifts the bare response and performs a baseline fitting such that the PA absorption before PMMA coating $\left(A_{\text {fit }}\right)$ fairly overlaps with one after PMMA coating ( $\left.A_{P M M A}\right)$. In Fig. 5(b), when the resonance frequency of the PA is exactly matched with absorption frequency of the $\mathrm{C}=\mathrm{O}$ band, absorption difference, and thus the sensitivity reaches its maximum value. Absorption difference decreases when 
the PA resonance is even slightly off from the carbonyl absorption frequency. The absorption difference varies between $13 \%$ and $33 \%$ of the PA resonance intensity. Even when the PA resonance is very off from the carbonyl absorption frequency, we still see a strong signal obtained from an only $10 \mathrm{~nm}$-thick PMMA film. The coupling between the plasmonic and PMMA vibrational modes results in spectral red-shift in the $\mathrm{C}=\mathrm{O}$ absorption dip from their usual spectral position at $1729 \mathrm{~cm}^{-1}$, as shown by the vertical red line in Fig. 5(b). When the PA supports a resonance frequency larger than $1729 \mathrm{~cm}^{-1}$ for $L_{1}$ values of between 1800 and $2200 \mathrm{~nm}$, the absorption dip corresponding to the $\mathrm{C}=\mathrm{O}$ band is observed at larger wavenumbers. When the PA supports a resonance frequency smaller than $1729 \mathrm{~cm}^{-1}$ for $L_{1}$ value of $2600 \mathrm{~nm}$, the absorption dip corresponding to the $\mathrm{C}=\mathrm{O}$ band is observed at smaller wavenumbers. When $L_{1}=2400 \mathrm{~nm}$, plasmonic resonance and the carbonyl absorption band are almost aligned. In Fig. 5(d), when $L_{5}=790 \mathrm{~nm}$, $1.5 \%$ absorption difference is obtained for the $-\mathrm{CH}_{3}$ asymmetric band stretch at $2952 \mathrm{~cm}^{-1}$. For other lengths of $L_{5}$, absorption differences at the $-\mathrm{CH}_{3}$ asymmetric band stretch frequency is not significant.

\section{Conclusion}

In conclusion, we experimentally presented a narrow band multi-resonant perfect absorber operating in the mid-IR range for the first time and demonstrated its usage for surface enhanced infrared absorption spectroscopy. Firstly, we performed theoretical analysis to investigate the physical origin of the dual-resonant behavior with strong near-field intensity enhancement distributions. Then, we obtained a fine-tuning mechanism to control the spectral response of the PAs through the geometrical parameters. Finally, we fabricated the numerically designated PAs and observed that the fabricated systems show similar optical responses compared to the calculations. In addition, we realized that the fabricated PAs actually show narrower resonance bands compared to the simulations or monolayer plasmonic nanoantennas. We also used the fabricated PAs to experimentally detect molecular fingerprints obtained from a $10 \mathrm{~nm}$-thick PMMA film. The SEIRA spectroscopy resulted in $33 \%$ intensity change on the PA resonance, which shows the sensitivity improvement obtained from narrow band resonances in comparison to monolayer plasmonic antennas. Fine tuning of the narrow-band PAs with high quality factor resonances allowed us to exactly match the relevant vibrational molecular modes. Thus, the presented narrow band multi-mode PA system is a promising probe to study very thin protein-lipid combined systems, such as live cell-membranes.

\section{Declaration of Competing Interest}

The authors declare that they have no known competing financial interests or personal relationships that could have appeared to influence the work reported in this paper.

\section{Acknowledgements}

Semih Korkmaz acknowledges support from The Council of Higher Education of Turkey100/2000 Doctoral Scholarship Program. Serap Aksu acknowledges support from the AXA Research Fund, EMBO Installation Grant 4155 and TUBITAK Grant 118F422.

\section{Appendix A. Supplementary data}

Supplementary material related to this article can be found, in the online version, at doi:https://doi.org/10.1016/j.sna.2019. 111757.

\section{References}

[1] Y. Ozaki, Recent advances in molecular spectroscopy of electronic and vibrational transitions in condensed phase and its application to chemistry, Bull. Chem. Soc. Jpn. 92 (2019) 629-654, http://dx.doi.org/10.1246/bcsj. 20180319.

[2] T. Jovanović, D. Koruga, A. Mitrović, D. Stamenković, G. Dević, IR and UV/VIS spectroscopic characterization of the higher fullerene $C_{76}-D_{2}$ for its quantitative and qualitative determination, J. Nanomater. 2018 (2018) 6862710, http://dx.doi.org/10.1155/2018/6862710.

[3] R. Iwamoto, R. Harui, FT-IR/NIR spectroscopic study of the hydrogen-bonding of $\mathrm{CH}$ to water in 1-Fluoroheptane, Bull. Chem. Soc. Jpn. 91 (2018) 1267-1274 http://dx.doi.org/10.1246/bcsj.20180063.

[4] J. Chalmers, P. Griffiths, Handbook of Vibrational Spectroscopy Volume V, Wiley, 1998.

[5] A.E. Cetin, D. Etezadi, H. Altug, Accessible nearfields by nanoantennas on nanopedestals for ultrasensitive vibrational spectroscopy, Adv. Opt. Mater. 2 (2014) 866-872, http://dx.doi.org/10.1002/adom.201400171.

[6] R. Adato, S. Aksu, H. Altug, Engineering mid-infrared nanoantennas for surface enhanced infrared absorption spectroscopy, Mater. Today 18 (2015) 436-446, http://dx.doi.org/10.1016/j.mattod.2015.03.001.

[7] D. Etezadi, J.B. Warner IV, F.S. Ruggeri, G. Dietler, H.A. Lashuel, H. Altug, Nanoplasmonic mid-infrared biosensor for in vitro protein secondary structure detection, Light: sci. Appl. 6 (2017) 17029, http://dx.doi.org/10. 1038/lsa.2017.29.

[8] D. Rodrigo, A. Tittl, A. John-Herpin, O. Limaj, H. Altug, Self-similar multiresonant nanoantenna arrays for sensing from near-to mid-infrared, ACS Photonics 5 (2018) 4903-4911, http://dx.doi.org/10.1021/acsphotonics. 8b01050.

[9] B. Cerjan, X. Yang, P. Nordlander, N.J. Halas, Asymmetric aluminum antennas for self-calibrating surface-enhanced infrared absorption spectroscopy, ACS Photonics 3 (2016) 354-360, http://dx.doi.org/10.1021/acsphotonics. $6 \mathrm{~b} 00024$.

[10] A.E. Cetin, S. Korkmaz, H. Durmaz, E. Aslan, S. Kaya, R. Paiella, M. Turkmen, Quantification of multiple molecular fingerprints by dual resonant perfect absorber, Adv. Opt. Mater. 4 (2016) 1274-1280, http://dx.doi.org/10.1002/ adom.201600305.

[11] O. Limaj, D. Etezadi, N.J. Wittenberg, D. Rodrigo, D. Yoo, S.H. Oh, H. Altug, Infrared plasmonic biosensor for real-time and label-free monitoring of lipid membranes, Nano Lett. 16 (2016) 1502-1508, http://dx.doi.org/10.1021/acs. nanolett.5b05316.

[12] L.V. Brown, K. Zhao, N. King, H. Sobhani, P. Nordlander, N.J. Halas, Surface-enhanced infrared absorption using individual cross antennas tailored to chemical moieties, J. Am. Chem. Soc. 135 (2013) 3688-3695, http://dx.doi.org/10.1021/ja312694g.

[13] D. Rodrigo, A. Tittl, N. Ait-Bouziad, A. John-Herpin, O. Limaj, C. Kelly, D. Yoo, N.J. Wittenberg, S.-H. Oh, H.A. Lashuel, H. Altug, Resolving molecule-specific information in dynamic lipid membrane processes with multi-resonant infrared metasurfaces, Nature Commun. 9 (2018) 2160, http://dx.doi.org/10. 1038/s41467-018-04594-X.

[14] L.V. Brown, X. Yang, K. Zhao, B.Y. Zheng, P. Nordlander, N.J. Halas, Fan-shaped gold nanoantennas above reflective substrates for surface-enhanced infrared absorption (SEIRA), Nano Lett. 15 (2015) 1272-1280, http://dx.doi.org/10. 1021/nl504455s.

[15] M. Osawa, K.I. Ataka, K. Yoshii, Y. Nishikawa, Surface-enhanced infrared spectroscopy: the origin of the absorption enhancement and band selection rule in the infrared spectra of molecules adsorbed on fine metal particles, Appl. Spectrosc. 47 (1993) 1497-1502, http://dx.doi.org/10.1366/ 0003702934067478

[16] Z. Li, S. Butun, K. Aydin, Ultranarrow band absorbers based on surface lattice resonances in nanostructured metal surfaces, ACS Nano 8 (2014) 8242-8248, http://dx.doi.org/10.1021/nn502617t.

[17] S. Chen, Z. Chen, J. Liu, J. Cheng, Y. Zhou, L. Xiao, K. Chen, Ultra-narrow band mid-infrared perfect absorber based on hybrid dielectric metasurface, Nanomaterials 9 (2019) 1350, http://dx.doi.org/10.3390/nano9101350.

[18] Z. Yong, S. Zhang, C. Gong, S. He, Narrow band perfect absorber for maximum localized magnetic and electric field enhancement and sensing applications, Sci. Rep. 6 (2016) 24063, http://dx.doi.org/10.1038/srep24063.

[19] N.I. Landy, S. Sajuyigbe, J.J. Mock, D.R. Smith, W.J. Padilla, Perfect metamaterial absorber, Phys. Rev. Lett. 100 (2008) 207402, http://dx.doi.org/ 10.1103/PhysRevLett.100.207402.

[20] N. Liu, M. Mesch, T. Weiss, M. Hentschel, H. Giessen, Infrared perfect absorber and its application as plasmonic sensor, Nano Lett. 10 (2010) 2342-2348, http://dx.doi.org/10.1021/n19041033.

[21] A. Tittl, P. Mai, R. Taubert, D. Dregely, N. Liu, H. Giessen, Palladium-based plasmonic perfect absorber in the visible wavelength range and its application to hydrogen sensing, Nano Lett. 11 (2011) 4366-4369, http://dx. doi.org/10.1021/nl202489g.

[22] E. Aslan, S. Kaya, E. Aslan, S. Korkmaz, O.G. Saracoglu, M. Turkmen, Polarization insensitive plasmonic perfect absorber with coupled antisymmetric nanorod array, Sens. Actuators B Chem. 243 (2017) 617-625, http://dx.doi.org/10.1016/j.snb.2016.12.030.

[23] E. Aslan, E. Aslan, M. Turkmen, O.G. Saracoglu, Experimental and numerical characterization of a mid-infrared plasmonic perfect absorber for dual-band 
enhanced vibrational spectroscopy, Opt. Mater. 73 (2017) 213-222, http://dx doi.org/10.1016/j.optmat.2017.08.023.

[24] J.A. Mason, G. Allen, V.A. Podolskiy, D. Wasserman, Strong coupling of molecular and mid-infrared perfect absorber resonances, IEEE Photonics Technol. Lett. 24 (2012) 31-33, http://dx.doi.org/10.1109/LPT.2011.2171942.

[25] C. Wu, A.B. Khanikaev, R. Adato, N. Arju, A.A. Yanik, H. Altug, G. Shvets, Fano-resonant asymmetric metamaterials for ultrasensitive spectroscopy and identification of molecular monolayers, Nat. Mater. 11 (2012) 69-75, http:// dx.doi.org/10.1038/nmat3161.

[26] A.E. Cetin, H. Altug, Fano resonant ring/disk plasmonic nanocavities on conducting substrates for advanced biosensing, ACS Nano 6 (2012) 9989-9995, http://dx.doi.org/10.1021/nn303643w.

[27] J.B. Lassiter, H. Sobhani, J.A. Fan, J. Kundu, F. Capasso, P. Nordlander, N.J. Halas, Fano resonances in plasmonic nanoclusters: geometrical and chemical tunability, Nano Lett. 10 (2010) 3184-3189, http://dx.doi.org/10.1021/ nl102108u.

[28] B. Luk'yanchuk, N.I. Zheludev, S.A. Maier, N.J. Halas, P. Nordlander, H. Giessen, C.T. Chong, Fano resonance in plasmonic nanostructures and metamaterials, Nat. Mater. 9 (2010) 707-715, http://dx.doi.org/10.1038/nmat2810.

[29] W. Klöpffer, Introduction to polymer spectroscopy, vol. VII, Springer Science \& Business Media, 2012

[30] Finite-Difference-Time-Domain Package, Lumerical FDTD Solutions, 2019 https://www.lumerical.com.

[31] K. Chen, R. Adato, H. Altug, Dual-band perfect absorber for multispectral plasmon-enhanced infrared spectroscopy, ACS Nano 6 (2012) 7998-8006, http://dx.doi.org/10.1021/nn3026468.

[32] H. Durmaz, Y. Li, A.E. Cetin, A multiple-band perfect absorber for SEIRA applications, Sens. Actuators, B 275 (2018) 174-179, http://dx.doi.org/10. 1016/j.snb.2018.08.053.

[33] E.D. Palik, Handbook of Optical Constants of Solids Volume III, Academic Press, 1998.

[34] M.J. Dodge, Refractive properties of magnesium fluoride, Appl. Opt. 23 (1984) 1980-1985, http://dx.doi.org/10.1364/AO.23.001980.

[35] R. Alaee, M. Albooyeh, C. Rockstuhl, Theory of metasurface based perfect absorbers, J. Phys. D Appl. Phys. 50 (2017) 503002, http://dx.doi.org/10.1088/ 1361-6463/aa94a8.

[36] R. Alaee, C. Menzel, C. Rockstuhl, F. Lederer, Perfect absorbers on curved surfaces and their potential applications, Opt. Express 20 (2012) 18370-18376, http://dx.doi.org/10.1364/OE.20.018370.

[37] J.R. Piper, V. Liu, S. Fan, Total absorption by degenerate critical coupling, Appl. Phys. Lett. 104 (2014) 251110, http://dx.doi.org/10.1063/1.4885517.

[38] C. Wu, B. Neuner III., G. Shvets, J. John, A. Milder, B. Zollars, S. Savoy, Large-area wide-angle spectrally selective plasmonic absorber, Phys. Rev. B 84 (2011) 075102, http://dx.doi.org/10.1103/PhysRevB.84.075102.

[39] W. Withayachumnankul, C. M. Shah, C. Fumeaux, S. Y. U. Benjamin, W. J. Padilla, M. Bhaskaran, D. Abbott, S. Sriram, Plasmonic resonance toward terahertz perfect absorbers, ACS Photonics 1 (2104) 625-630, https://doi.org/10.1021/ph500110t.

[40] H. Noh, Y. Chong, A.D. Stone, H. Cao, Perfect coupling of light to surface plasmons by coherent absorption, Phys. Rev. Lett. 108 (2012) 186805, http:// dx.doi.org/10.1103/PhysRevLett.108.186805.

[41] S. Kang, Z. Qian, V. Rajaram, S.D. Calisgan, A. Alù, M. Rinaldi, Ultra-narrowband metamaterial absorbers for high spectral resolution infrared spectroscopy, Adv. Opt. Mater. 7 (2019) 1801236, http://dx.doi.org/ 10.1002/adom.201801236.

[42] F. Le, D.W. Brandl, Y.A. Urzhumov, H. Wang, J. Kundu, N.J. Halas, J. Aizpurua, P. Nordlander, Metallic nanoparticle arrays: a common substrate for both surface-enhanced Raman scattering and surface-enhanced infrared absorption, ACS Nano 2 (2008) 707-718, http://dx.doi.org/10.1021/ nn800047e.

[43] Y. Guo, L. Yan, W. Pan, B. Luo, X. Zhang, X. Luo, Misalignments among stacked layers of metamaterial terahertz absorbers, Front. Optoelectron. 7 (2014) 53-58, http://dx.doi.org/10.1007/s12200-013-0380-4.

[44] R. Adato, A.A. Yanik, J.J. Amsden, D.L. Kaplan, F.G. Omenetto, M.K. Hong, S. Erramilli, H. Altug, Ultrasensitive vibrational spectroscopy of protein monolayers with plasmonic nanoantenna arrays, Proc. Natl. Acad. Sci. U. S. A. 106 (2009) 19227-19232, http://dx.doi.org/10.1073/pnas.0907459106.

[45] S. Aksu, A.E. Cetin, R. Adato, H. Altug, Plasmonically enhanced vibrational biospectroscopy using low-cost infrared antenna arrays by nanostencil lithography, Adv. Opt. Mater. 1 (2013) 798-803, http://dx.doi.org/10.1002/ adom.201300133.

\section{Biographies}

Semih Korkmaz received his B.S. and M.S. degrees from Erciyes University, Kayseri, Turkey, both in the Department of Electrical and Electronics Engineering in 2013 and 2015, respectively. He has been currently working toward the Ph.D. degree in the Department of Electrical and Electronics Engineering, Erciyes University, Kayseri, Turkey since 2015. He is also a visiting researcher at Institute of Materials Science and Nanotechnology, Bilkent University, Ankara, Turkey. His current research interests include nanoplasmonics, perfect absorbers for biosensing applications. He is a doctoral scholarship supported by The Council of Higher Education of Turkey (Program Code: 100/2000)

Dr. Mustafa Turkmen is an Associate Professor in the Electrical and Electronics Engineering Department at Erciyes University, Kayseri, Turkey. He received his M.S. and Ph.D. degrees from Erciyes University, both in Electrical and Electronics Engineering, in 2003 and 2009, respectively. Dr. Turkmen worked in the Electrical and Computer Engineering Department, in Boston University, Boston, MA, USA as a research Assistant Professor between 2009 and 2012. His current research interests include microwave printed circuits, and patch antennas for modern wireless communication systems, metamaterials and plasmonic nanoantenna arrays for biosensing applications.

Dr. Serap Aksu received her B.S. degree from Sabanci University, Istanbul, Turkey, in the Department of Materials Science and Engineering in 2008 and Ph.D. degree from Boston University, Boston, MA, USA, in the Department of Materials Science and Engineering, Photonics Center in 2013. She worked as a Postdoctoral Fellow at ETH Zurich between 2014 and 2016. She won incoming researcher fellowship from The Scientific and Technical Research Council of Turkey (TUBITAK) in 2017. Now, she is an Assistant Professor in the Physics Department at Koc University, Istanbul, Turkey. She is the recipient of EMBO Installation Grant (2019) and AXA research Fund (2019). 